\title{
Analytical and numerical approach to defining the stability region of a dynamical system
}

\author{
M. NEŠTICKÝ, O. PALUMBÍNY AND G. MICHALČONOK
}

\begin{abstract}
The paper presents two different approaches to estimating the region of stability of differential equation. Estimation of the region of stability is an essential practice in relation to control of the dynamical system. In this paper the objects of examination are differential equations with quasi-derivation. The equations have features that do not allow the application of classical methods for establishing stability. The goal is to compare the results of an analytic approach using Lyapunov method and computer simulation using a numerical method. The brief description of both methods are introduced and graphical results are presented and compared.
\end{abstract}

Mathematics Subject Classification 2000: 65L05, 34D20

Keywords: dynamical system, numerical analysis, stability

\section{INTRODUCTION}

Theory of dynamical systems is applicable across the entire fields of technical science. The mathematic relations discovered during examining a problem can be usually applied even in seemingly unrelated problems. Therefore, it is relevant to study the characteristic features of a certain class of equations in general. It can reveal a characteristic of physical systems linked with these equations. Systems described by differential equation with quasi-derivatives bring new challenges, which cannot be covered by theory using only classical derivations. In particular, many authors investigate the oscillatory and asymptotic properties of solutions of differential equations of the $n$-th order with quasi-derivation [Skerlik; Rovder; Soltes; Mojsej]. Stability theory plays central important role in system theory and engineering. Therefore, research on stability systems expressed by differential equations with the quasi-derivations is highly current.

Powerful and well-known tools for establishing the stability criteria exist for linear systems [Parks; Hahn]. Nowadays, the challenge is to establish similar criteria for the dynamical systems which are described by the various differential equations and not only by the linear fashion.

Computer simulation and numerical methods in [Ahmed; Morzynski; Sutova] 
represent useful tools for stability analysis, but a better understanding of how the choice of a particular formula influences not only computing but also the representation of the obtained results is needed.

Purpose of this paper is to compare two different approaches to solve the same problem. Stability criteria derived utilizing an analytic method with results obtained by a numerical modeling of the system has been confronted. In order to achieve comparable results, a specific simulation was proposed and it was performed with graphically illustrated results.

This paper deals with a criterion of an asymptotic stability in Liapunov sense for a null solution of the autonomous third-order differential equations with the quasiderivatives

$$
L_{4} y=f\left(L_{0} y, L_{1} y, L_{2} y, L_{3} y\right)
$$

where (the prime means a derivative owing to the variable)

$$
\begin{aligned}
& L_{0} y(t)=y(t), \\
& L_{1} y(t)=p_{1}(t)\left(L_{0} y(t)\right)^{\prime}, \\
& L_{2} y(t)=p_{2}(t)\left(L_{1} y(t)\right)^{\prime}, \\
& L_{3} y(t)=p_{3}(t)\left(L_{2} y(t)\right)^{\prime}, \\
& L_{4} y(t)=\left(L_{3} y(t)\right)^{\prime} .
\end{aligned}
$$

$p_{i}(t), i=1,2,3$ are real-valued continuous functions which enable an user a better control of a considered processes described by the equation (1). The terms $L_{k} y(t), k=1,2,3$ are the $k$-th quasi derivative of a function $y(t)$. We note that the function $p_{i}(t), i=1,2,3$ is not, in general, assumed to be differentiable and we can use stability criteria derived for differential equation with classic derivatives.

\section{LYAPUNOV INDIRECT METHOD}

Single differential equation of the $n$th order can be reduced to a system of $n$ firstorder equations by a simple change in notation. Equivalent system of the equation can be considered (1) as follows

$$
\begin{aligned}
& y_{1}^{\prime}=y_{2} / p_{1}(t) \\
& y_{2}^{\prime}=y_{3} / p_{2}(t) \\
& y_{3}^{\prime}=y_{4} / p_{3}(t) \\
& y_{4}^{\prime}=f\left(y_{1}, y_{2}, y_{3}, y_{4}\right) .
\end{aligned}
$$


Assertion which was proven in [Palumbiny] is introduced. The authors determined a detailed procedure for deriving these formulas. Results arising from this established stability criteria were elaborated.

Let the differential equation be considered as(3):

$$
\lim _{t \rightarrow \infty} p_{i}(t)=a_{i}>0, i=1,2,3
$$

If the entire real part of zeros of

$$
s^{4}-\frac{\partial f}{\partial y_{4}}(\mathbf{0}) s^{3}-\frac{\partial f}{\partial y_{3}}(\mathbf{0}) \frac{1}{a_{3}} s^{2}-\frac{\partial f}{\partial y_{2}}(\mathbf{0}) \frac{1}{a_{2} a_{3}} s-\frac{\partial f}{\partial y_{1}}(\mathbf{0}) \frac{1}{a_{1} a_{2} a_{3}}
$$

is negative, then the null solution 0 is asymptotically stable in Liapunov sense.

Lyapunov indirect method was used to derive the formulas. The polynomial (5) is a characteristic polynomial of the linearized matrix of the system (3). The indirect method of Lyapunov uses the linearization of a system to determine the local stability of the solution of the system. Without a loss of generality it is assumed, that the function 0 is a solution of equation (1). Function 0 is a solution of (1) if and only if a vector $(0,0,0,0)$ is a solution of $(3)$. This means, that the vector $(0,0,0,0)$ is an equilibrium point of the equation (3).

To determine the criteria of stability of our system, the Routh-Hurwitz criterion for fourth-degree polynomials has been applied [Parks]. Following from the mentioned criteria, the null solution 0 of equation (1) is asymptotically stable in Lyapunov sense if it holds that

$$
\begin{aligned}
& \frac{\partial f}{\partial y_{1}}<0, \\
& \frac{\partial f}{\partial y_{2}}<0, \\
& \frac{\partial f}{\partial y_{2}} \frac{\partial f}{\partial y_{3}} \frac{1}{a_{2} a_{3}^{2}}>\frac{\partial f}{\partial y_{1}} \frac{\partial f}{\partial y_{4}} \frac{1}{a_{1} a_{2} a_{3}}, \\
& \frac{\partial f}{\partial y_{1}} \frac{\partial f}{\partial y_{4}} \frac{1}{a_{1} a_{2} a_{3}}>\frac{\partial f}{\partial y_{2}} \frac{\partial f}{\partial y_{3}} \frac{\partial f}{\partial y_{4}} \frac{1}{a_{2} a_{3}^{2}}+\frac{\partial f^{2}}{\partial y_{2}} \frac{1}{a_{3}^{2}} .
\end{aligned}
$$

\subsection{Region of stability}

The presented criterion can be used for stabilizing the system. The parameters of the differential equations are based on the features of the observed systems. In practical use, it is not possible to change the value of most of these parameters. Only some parameters can be changed in limited range. If these parameters can be set such 
that (6), then the system can be stabilized. This procedure has been used to obtain important information about the system without the need of solving the differential equation.

Let us consider equation (1), where

$$
L_{4} y=-4 y-10 L_{1} y-10 L_{2}-5 L_{3} y \text {. }
$$

By inputting $p_{i}(t)=1, i=1,2,3$ a differential equation with classic derivatives was obtained. The characteristic polynomial of this equation can be expressed in the form $h(s)=(s+1)(s+2)(s+1+i)(s+1-i)$. This immediately determines that all zeros have negative real parts. Thus, the equation has a stable zero solution. For our purpose, it is interesting to observe how the varying values of the functions $p_{i}(t), i=1,2,3$ affect the qualitative features of the dynamical equation.

Competent system of the equation (7) is

$$
\begin{aligned}
& y_{1}^{\prime}=y_{2} / p_{1}(t) \\
& y_{2}^{\prime}=y_{3} / p_{2}(t) \\
& y_{3}^{\prime}=y_{4} / p_{3}(t) \\
& y_{4}^{\prime}=f\left(-4 y_{1}-10 y_{2}-10 y_{3}-5 y_{4}\right)
\end{aligned}
$$

Now, let $p_{1}=u_{1}+\frac{1}{t}, p_{2}=u_{2}+\frac{2}{t}, p_{3}=u_{3}+\frac{3}{t}$, where $u_{k}$ are real constant numbers. According to (4) $a_{i}=u_{i}, i=1,2,3$ and partial derivatives of the function $f$ are of the values $\frac{\partial f}{\partial y_{1}}=-4, \frac{\partial f}{\partial y_{2}}=-10, \frac{\partial f}{\partial y_{3}}=-10, \frac{\partial f}{\partial y_{4}}=-5$. If the constants are inserted into the formulas for stability criteria (6) of our system, a set of an inequalities which represent region of stability of the equation (1) is obtained. In the Figure 1 is a graphical representation of the region of stability.

\section{COMPUTER SIMULATION}

Other procedure on how to obtain more information is using a numerical method. If all that is needed is one solution of a differential equation through a particular point, then it can begin at the point, compute local direction, and then compute the slopes at a few nearby points. This process would always draw only those slopes, which are near, where finding the solution is expected. A computer may be useful in such a case. Accurate and sophisticated methods are readily available in various mathematical computer environments. MATLAB's standard solver for ordinary differential equation is defined by the function ode45. This function 


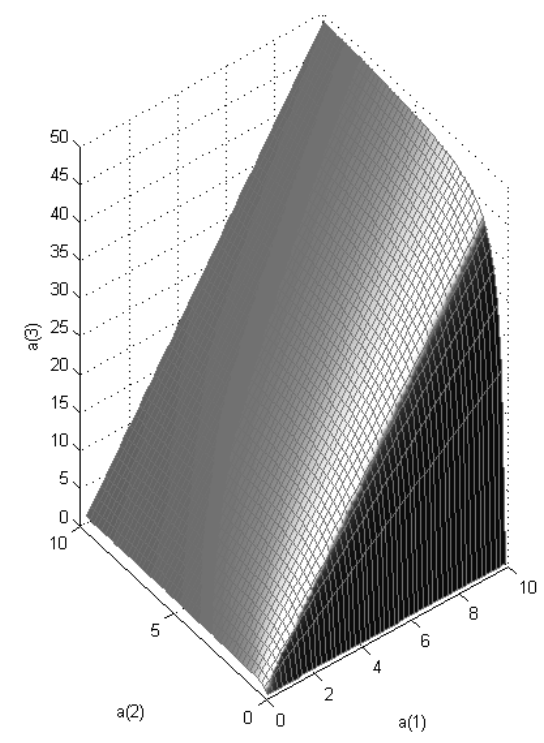

Fig. 1. Region of stability based on an analytical solution

implements a Runge-Kutta method with a variable time step for efficient computation. ode 45 can only solve a first order differential equation, therefore set of equations (8) are computed. However, a result is very similar to the reality, the input information must be complete, including initial conditions. It can be seen, whether the particular system is stable or not, but it is not clear how the system must change in order to stabilize itself.

It is possible to define a region of stability for the differential equation with a varying parameters by computer simulation, but this can be more than a difficult task for an equation with three or more varying parameters and graphical representation in 3D probably will not fulfill the expectations. Therefore a system (8) where $p_{1}=1+\frac{1}{t}, p_{2}=u_{2}+\frac{2}{t}, p_{3}=u_{3}+\frac{3}{t}$ was studied. It is a similar case as the one mentioned above, but $u_{1}=1$ and graphical representation of the region of stability is now represented by a cross section of the object on the Figure 1 in the place where $u_{1}=1$. Figure 2 shows the cross section. Black color represent the stable system and white color represent the instable system.

Lyapunov type of stability is related to the trajectory of solutions near to a point of 


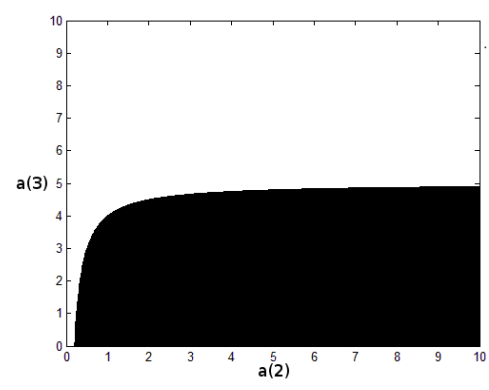

Fig. 2. Cross section of the analytical solution

equilibrium (the null solution $(0,0,0,0)$ ). If the solutions that begin near an equilibrium point stay near infinity, then the equilibrium point is Lyapunov stable. For studying these phenomena by a computer simulation a set of experiments was proposed. The Matlab's function ode45 provides only particular solution. A lot of simulations has to be performed to obtain a pattern which can be compared with the analytical method introduced above and can verify the results. Vector $(0,0,0,1)$ was an initial condition for every simulation, but parameters $u_{2}$ and $u_{3}$ were randomly varying on interval from zero to ten. Three sets of simulations were performed. The first one was interrupted at time $t=50$, the second at $t=100$ and the third at $t=200$. The computing time was the same for each set of simulations. Results of the simulations are represented by three figures. If function ode 45 finished calculating the particular solution, then the magnitude of the last vector of the solution indicates whether the solution converges to equilibrium point or diverges. We use a vector norm of the form $|\mathbf{y}|=\sqrt{y_{1}^{2}+y_{2}^{2}+y_{3}^{2}+y_{4}^{2}}$ to determine magnitude of vector. If this magnitude was equal to zero, then the equation is considered as a stable. The black point is located on the figure. The place on the figure corresponds to the parameters of equation, which was calculated. The brightness of a point exponentially increases with the increase in distance between norm of the solution and the equilibrium point. Scattering of these points creates patterns, which are shown in Figure 3.

As we can see in the Figure 2, analytical results provide sharp boundaries between stable and unstable systems. However, equations define that stable system is stabilized in infinite time. In practice, however, we need to know what the state of the particular system is after a certain time. Such information is provided by numerical analysis. From the Figure 3, it can be determined for various parameters whether the system 

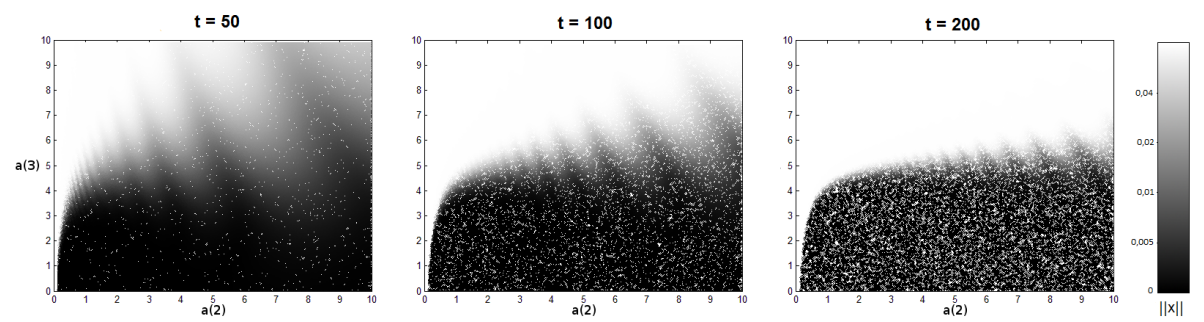

Fig. 3. Computer simulation of region of stability

solution went to zero, to infinity or is moving less or more near the origin. Moreover, in these figures, certain shapes are presented that are caused by the periodicity of the solution, which can reduce the accuracy, but on the other hand, new important information can be obtained.

\section{CONCLUSION}

The foregoing results can be used for ordinary differential equations with quasiderivatives. Especially, when the functions $p_{i}(t), i=1,2,3$ are not differentiable on a considered interval, where classical stability criteria cannot be used. Beside the establishment of stability criteria, a focus was also made on their validation. Method to compare analytical results with numerical results was introduced. It appears that by applying this method not only the region of stability but also the next qualitative features of the solution dependent on varying parameters was obtained.

\section{ACKNOWLEDGEMENTS}

This publication is the result of implementation of the project 1602 Development of integrated hierarchical mechatronic systems by Functional, Reliable, and Security Aspects of "Industry 4.0" supported by the (IVP) MTF STU 2017

The authors would like to thank for financial contribution from the STU Grant scheme for Support of Young Researchers.

\section{REFERENCES}

A. Skerlik. Criteria of property $A$ for third order superlinear differential equations. Mathematica Slovaca 43(2):171-183, 1993.

J. Rovder. Comparison theorems for third-order linear differential equations. Bulletin of the Institute of Mathematics Academia Sinica 19:43-52, 1991. 
V. Soltes. Oscillatory properties of solutions of a fourth-order nonlinear differential equation. Mathematica Slovaca 29(1):73-82, 1979.

I. Mojsej \& J. Ohriska. On solutions of third order nonlinear differential equations. Open Mathematics 4(3):46-63, 2006.

P. C. Parks. A new proof of the Routh-Hurwitz stability criterion using the second method of Liapunov. In Mathematical Proceedings of the Cambridge Philosophical Society 58(04):694-702,1962

W. Hahn. Stability of motion (Vol. 138) Berlin: Springer, 1967.

E. Ahmed, A. M. A. El-Sayed \& H.A. El-Saka. Equilibrium points, stability and numerical solutions of fractional-order predator-prey and rabies models. Journal of Mathematical Analysis and Applications 325(1):542-553, 2007.

M. Morzynski \& F. Thiele. Numerical stability analysis of a flow about a cylinder. Zeitschrift Angewandte Mathematik und Mechanik 71:424, 1991.

Z. Sutova \& R. Vrabel Numerical Investigation of Initial Condition and Singular Perturbation Parameter Value Influence on the Dynamical System Oscillatory Behaviour. Intelligent Networking and Collaborative Systems (INCoS), 2016 International Conference on. IEEE, 2016.

O. Palumbiny \& M. Nesticky Criteria for asymptotic stability of fourth-order nonlinear differential equations with quasi-derivatives. Advances in Circuits, Systems, Signal Processing and Telecommunications, 2015.

\author{
Martin Neštický \\ Institute of Applied, Automation and Mechatronics, \\ Slovak University of Technology in Bratislava, 91724 Trnava \\ Email: martin.nesticky@stuba.sk
}

\author{
Oleg Palumbíny \\ Institute of Applied, Automation and Mechatronics, \\ Slovak University of Technology in Bratislava, 91724 Trnava \\ Email: oleg.palumbiny@stuba.sk
}

\author{
German Michalčonok \\ Institute of Applied, Automation and Mechatronics, \\ Slovak University of Technology in Bratislava, 91724 Trnava \\ Email: german.michalconok@stuba.sk
}

